\title{
Epidemiologic Evaluation of Central Nervous System Sarcomas in Iran: A 2009-2014 Survey
}

\section{Seddighi AS ${ }^{1}$, Nikouei $A^{2}$, Seddighi $A^{1 *}$, Akbari $M^{3}$, Arjmand $Y^{2}$, Ommi $D^{4}$, Zali $A^{5}$ and Hosseini $\mathbf{M}^{2}$}

${ }^{1}$ Shohada Tajrish Comprehensive Neurosurgical Center of Excellence, Functional Neurosurgery Research Center of Shohad Tajrish Hospital, Shahid Beheshti University of Medical Sciences, Tehran, Iran

${ }^{2}$ GP Member of Shohada Tajrish Comprehensive

Neurosurgical Center of Excellence, Functional

Neurosurgery Research Center of Shohad Tajrish

Hospital, Shahid Beheshti University of Medical Sciences,

Tehran, Iran

${ }^{3}$ Cancer Research Center, Shohada Tajrish Hospital, Shahid Beheshti University of Medical Sciences, Tehran, Iran

${ }^{4}$ Shohada Tajrish Comprehensive Neurosurgical Center of Excellence, Functional Neurosurgery Research Center of Shohad Tajrish Hospital, Shahid Beheshti University of Medical Sciences, Tehran, Iran

${ }^{5}$ Shohada Tajrish Comprehensive Neurosurgical Center of Excellence, Functional Neurosurgery Research Center of Shohad Tajrish Hospital, Shahid Beheshti University of Medical Sciences, Tehran, Iran

*Corresponding author: Afsoun Seddighi, Associate Prof of Neurosurgery. Shohada Tajrish Comprehensive Neurosurgical Center of Excellence, Functional

Neurosurgery Research Center of Shohad Tajrish

Hospital, Shahid Beheshti University of Medical Sciences, Tehran, Iran

Received: April 22, 2021; Accepted: May 13, 2021;

Published: May 20, 2021

\begin{abstract}
Sarcomas rise from mesenchymal layers and may transform to variable malignant lesions in multiple organs systems. Although they are mostly localized, many of them have predilection of distant sites away from their primary origins and subsequent metastasis to different anatomical locations of body. Soft Tissue Sarcomas (STSs) represent one percent of adult and about $7 \%$ of pediatric malignancies. Although insignificant in number, these tumors may complicate patient's overall health status compared with other malignancies, because of their numerous subtypes and variable features which may pose an everlasting challenge to both clinical and surgical treatment strategies. Existence of these complications and a relatively small available data regarding Central Nervous System's (CNS) sarcomas, encouraged authors to conduct a retrospective study in a 6-year period to evaluate the specific epidemiological features of CNS sarcomas, including brain, spinal cord and meningeal layer sarcomas extracted from Iranian National Cancer Registry (INCR). Our study revealed CNS sarcoma's trend to affect males more than females, affecting them most in their midlife and a higher prevalence of brain involvement compared to spinal cord and meningeal layers. Also, we have provided detailed morphological features of the tumors, as well as patient's geographical distribution, with Northern parts of Iran have the lowest incidence rate (10.06\%). Moreover, our analysis of crude rate revealed lower Age Specific Incidence Rate (ASIR) of CNS Sarcomas than expected number of cases compared to standard world population by World Health Organization (WHO)'s ASIR as 0.035 person per 100000-years. Authors believe this report of epidemiological assessment of CNS sarcomas in Iran could act as a foundation for better understanding the underlying pathophysiological mechanism as it is the first survey in developing countries and selection of optimal treatment strategies based on improved understanding of these neoplasms.
\end{abstract}

Keywords: Sarcoma; Soft tissue sarcoma; Central nervous system; Epidemiologic study

\section{Introduction}

Being derived from Greek word sarx meaning flesh, sarcomas originates from transformed mesenchymal cells with numerous subtypes and various distribution pattern and prognosis [1]. Mentioned versatility coupled with its rarity, are two main factors responsible for restricted reports of sarcomas description throughout history, which account for current limited knowledge on appropriate management of different types of sarcomas [2,3]. The American Cancer Society's estimation of new bone and soft tissue sarcomas are 3500 and 12750 cases in the United States by the end of 2019 [4]. Considering the total estimate of new diagnosed cancer is 1762450 cases, bone and soft tissue sarcomas constitute about 0.2 and $0.7 \%$ of newly diagnosed malignancies respectively, making total cases of estimated new sarcomas roughly $1 \%$ of all new diagnosed cancers [57]. Following previous statements regarding description of sarcomas rarity, multiple studies suggest lifetime risk of development of this cancer as about $0.001 \%$ [8]. Among these tumors, Central Nervous System (CNS) sarcomas represent an extremely rare subgroup with less than $1 \%$ of all sarcomas, originating mostly as a result of distant metastasis, direct invasion to the brain parenchyma and radiation associated sarcomas in young individuals $[9,10]$. Undifferentiated sarcoma, fibrosarcoma and malignant fibrous histiocytoma were among the most commonly found subtypes of CNS sarcomas in the past decade [11]. In addition to previous reports of incidence rate of CNS sarcoma of 3 per 10 million person-years, primary Intracranial Sarcomas (IS) which were first described in 1929, have incidence rate from 0.1 to $4.3 \%$ based on multiple types of studies with variable definition of primary IS $[12,13]$. Inclusion of reticulum cell sarcoma, circumscribed sarcoma of cerebellum and hemangiopericytoma and report's inconsistency in unified definition of primary IS have contributed to this high value of incidence despite its rarity [14]. Since epidemiologic and descriptive studies are the cornerstones of enhanced understanding of these group of malignancies, authors decided to describe the epidemiologic aspect of CNS sarcomas and the prevalence of these challenging tumors in brain and spinal column and their subtypes in a 6-year period in Iran from 2009 to 2014.

\section{Methods}

In this retrospective study, authors have analyzed patients with confirmed histopathological study of CNS sarcomas in Iranian population. Patients with CNS sarcomas were identified from Iranian 
National Cancer Registry (INCR) from April 2009 to December 2014. Also, authors extracted population data of from Iranian National Cancer Registry. Patient's demographic data including age, sex, living environment and city, primary tumor location and histopathological subtype based on World Health Organization (WHO) classification system of sarcoma with confirmed diagnosis, were gathered and recorded. Patient's specific tumor data documented in INCR have been filed based on histopathological and radiological confirmation, with roughly 16700 documented sarcoma patients were analyzed in the same mentioned 6-year period. Due to recent changes of STSs classification system and ongoing updates, authors have reported both macroscopic and microscopic tumor description as tumor's morphology and its classification, respectively. Since some tumors have higher incident rate in specific geographical areas, we have analyzed the geographical distribution pattern of our patients to find a possible relevant "hot-zone" for CNS sarcomas in Iran, given that it may lead to extended research on potential environmental hazards and carcinogens. Also, authors performed statistical analysis using SPSS program version 22 to perform Age Specific Incidence Rate (ASIR) and its annual trend.

\section{Results}

In this study, authors have thoroughly examined the INCR's information in a period between 2009 to 2014, and analyzed 298 patients with confirmed diagnosis of CNS sarcomas, either of brain (primary IS) or spinal cord, out of the 16700 sarcoma patients. Our analysis revealed 180 males and 118 females with male to female ratio of 1.52 and a periodic prevalence of $1.78 \%$. Specific details of CNS sarcoma prevalence among both sexes is described in Table 1.

Also, authors have classified patients based on their presenting age on 3 main groups as 0-14 years as age range group I, 15-64 years as age range group II and 65-100 years as age range group III. Based on the results, 31 patients (10.4\%) were categorized as group I, 227 patients $(76.17 \%)$ as group II and 40 patients (13.42\%) as group III. Detailed age specifications are demonstrated on Table 2.

Authors deep further in statistical analysis and performed Age Specific Incidence Rate (ASIR) analysis of CNS sarcomas in complete study period demonstrated in Table 3. Our analysis revealed ASIR of CNS sarcoma as 0.396 per 100000 person years compared to 0.431 per 100000 person years of expected number cases based on Standard World Population from WHO, that indicate lower ASIR of our study compared to the latest with a new age range classification, as children and adolescent (0-19 years), young adults (20-34 years), old
Table 2: Age range specification of patients with CNS sarcomas.

\begin{tabular}{|c|c|c|c|c|c|c|}
\hline \multirow{2}{*}{$\begin{array}{l}\text { Age Range } \\
\text { (years) }\end{array}$} & \multicolumn{6}{|c|}{ Study Year } \\
\hline & 2009 & 2010 & 2011 & 2012 & 2013 & 2014 \\
\hline $0-4$ & 0 & 0 & 3 & 2 & 5 & 3 \\
\hline $5-9$ & 2 & 0 & 4 & 3 & 3 & 1 \\
\hline $10-14$ & 0 & 0 & 3 & 0 & 2 & 0 \\
\hline $15-19$ & 0 & 1 & 6 & 2 & 3 & 2 \\
\hline $20-24$ & 1 & 0 & 3 & 6 & 5 & 6 \\
\hline $25-29$ & 0 & 0 & 9 & 4 & 3 & 1 \\
\hline $30-34$ & 0 & 0 & 6 & 8 & 5 & 5 \\
\hline $35-39$ & 1 & 3 & 7 & 6 & 1 & 3 \\
\hline $40-44$ & 0 & 2 & 7 & 9 & 4 & 5 \\
\hline $45-49$ & 1 & 3 & 9 & 3 & 8 & 1 \\
\hline $50-54$ & 1 & 1 & 8 & 8 & 3 & 7 \\
\hline $55-59$ & 0 & 4 & 3 & 8 & 9 & 7 \\
\hline $60-64$ & 0 & 3 & 2 & 5 & 4 & 5 \\
\hline $65-69$ & 2 & 0 & 8 & 4 & 6 & 2 \\
\hline $70-74$ & 0 & 2 & 2 & 2 & 2 & 2 \\
\hline $75-79$ & 0 & 1 & 2 & 1 & 1 & 0 \\
\hline $80-100$ & 0 & 0 & 1 & 1 & 1 & 0 \\
\hline Sum & 8 & 20 & 83 & 72 & 65 & 50 \\
\hline
\end{tabular}

adults (35-59 years) and elderly (more than 65 years) based on WHO definitions (Table 4, Figure 1).

Given annual trend above state that elderly population are at the highest risk of CNS sarcoma development, while children and adolescents stands at the opposite edge with the lowest probability. On the other hand, clinical and surgical evaluations revealed the anatomical location of CNS sarcomas, reported as located in brain as 184 cases (61.7\%), followed by 101 cases $(33.8 \%)$ in the spine and 13 cases (4.3\%) located primarily on their meningeal layers. Authors have classified the studied lesions from histopathological point of view and have provided their grade based on World Health Organization (WHO)'s sarcoma scoring system (ICD-O). Tissue examination of CNS sarcomas revealed 10 cases (3.3\%) as grade 1 or adipocytic tumors, 14 cases $(4.6 \%)$, as grade 2 or fibroblastic/ myofibroblastic tumors, 15 cases as grade 3 or fibrohistiocytic tumors (5\%), 11 cases (3.6\%) as grade 4 or smooth muscle tumors and other 248 cases $(83.2 \%)$ as grade 9 or tumors of uncertain differentiation.

Table 1: Sarcoma distribution based on gender and CNS involvement.

\begin{tabular}{|c|c|c|c|c|c|c|}
\hline \multirow{2}{*}{ Year (CNS sarcoma patients) } & \multicolumn{3}{|c|}{ Brain } & \multicolumn{3}{|c|}{ Spine } \\
\hline & Male (percentage) & Female (percentage) & M:F ratio & Male (percentage) & Female (percentage) & M:F ratio \\
\hline $2009(8)$ & $6(75 \%)$ & $1(12.5 \%)$ & 6 & $1(12.5 \%)$ & 0 & 1 \\
\hline $2010(20)$ & $8(40 \%)$ & $10(50 \%)$ & 0.8 & $1(5 \%)$ & $1(5 \%)$ & 1 \\
\hline $2011(83)$ & $38(45.7 \%)$ & $25(30.1 \%)$ & 1.52 & $12(14.4 \%)$ & $8(9.6 \%)$ & 1.5 \\
\hline $2012(72)$ & $25(34.7 \%)$ & $14(19.4 \%)$ & 1.78 & $20(27.7 \%)$ & $13(18 \%)$ & 1.53 \\
\hline $2013(65)$ & 27 (41.5\%) & $16(24.6 \%)$ & 1.68 & $16(24.6 \%)$ & $6(9.2 \%)$ & 2.66 \\
\hline $2014(50)$ & $18(36 \%)$ & $15(30 \%)$ & 1.2 & $6(12 \%)$ & $11(22 \%)$ & 0.54 \\
\hline Total (298) & $122(40.9 \%)$ & $81(27.1 \%)$ & 1.5 & $56(18.7 \%)$ & $39(13 \%)$ & 1.43 \\
\hline
\end{tabular}

CNS: Central Nervous System, M: Male, F: Female. 
Table 3: Age Specific Incidence Rate of CNS sarcomas.

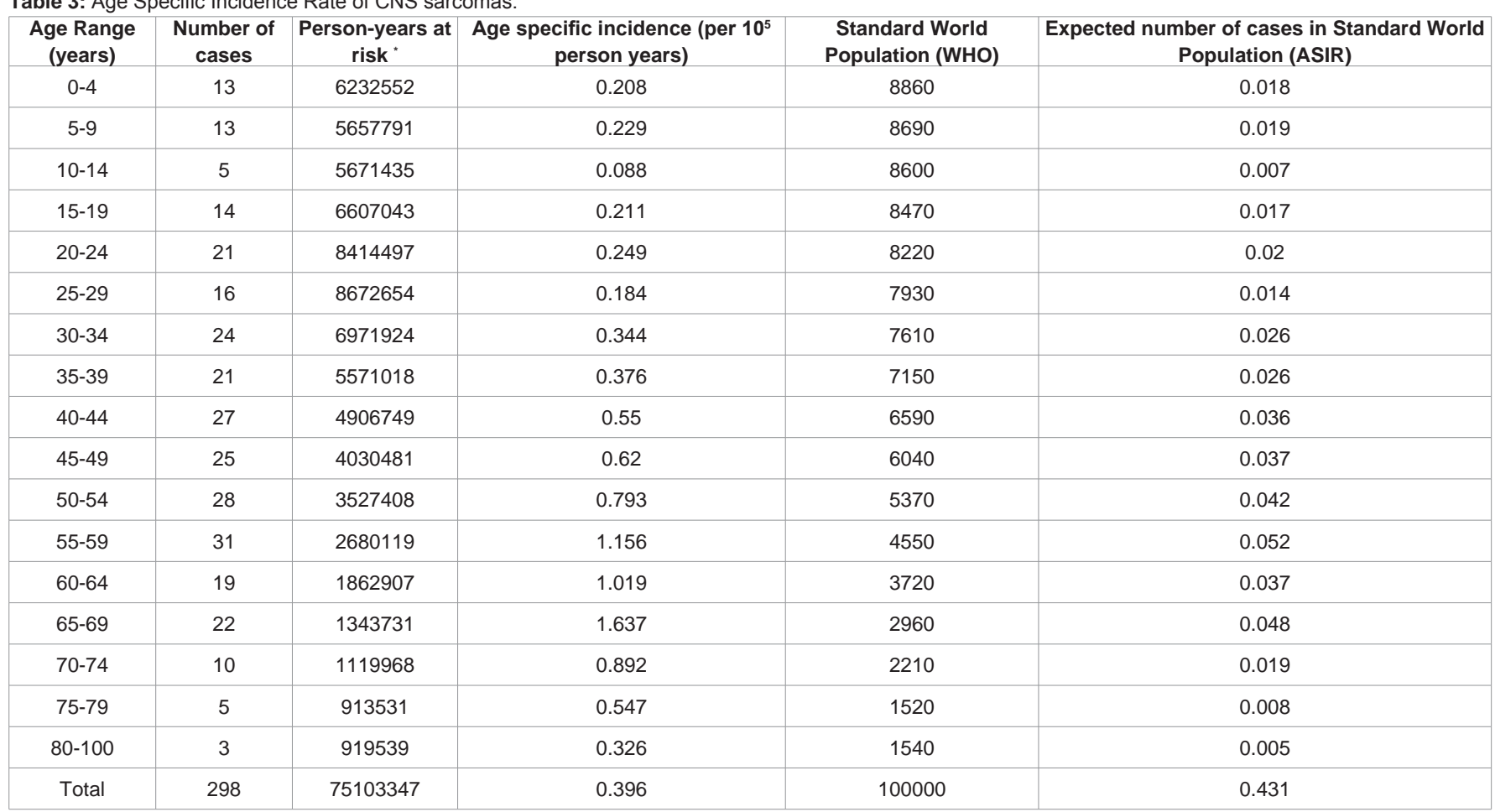

WHO: World Health Organization; ASIR: Age Specific Incidence Rate.

${ }^{*}$ Extracted from Statistical Center of Iran.

Table 5 contains details of morphological features of excised and studied lesions of our study sample. Also, our analysis regarding most encountered histopathological subtype of CNS sarcoma in each age group category which defined above, age group 1 (0-14 years) mainly consisted of sarcoma with mostly unspecified grade (22.5\%), followed by desmoplastic small round cell tumor with unspecified grade and gliosarcoma with mostly unspecified grade (12.9\% each). Also, gliosarcoma with mostly unspecified grade ranked first (18.9\%) in age group 2 (15-64 years), followed by chordoma with unspecified grade $(11.8 \%)$ and desmoplastic small round cell tumor with mostly unspecified grade (11\%). At the latest age group as group 3 (65-100 years), gliosarcoma with mostly unspecified grade (40\%) consist the major CNS sarcoma type, followed by hemangiopericytoma malignant with mostly grade $3(17.5 \%)$ and chordoma with unspecified grade (10\%) According to previous statements of possibility of geographical relevance to development of STSs, especially CNS sarcomas, we have assessed the distribution of our patients based on their living area and country's territories on Table 6.

\section{Discussion}

Based on histopathological classifications, subtypes of bone Table 4: Age Specific Incidence Rate of CNS sarcomas on annual basis.

\begin{tabular}{|l|c|c|c|c|c|c|}
\hline \multirow{2}{*}{\multicolumn{1}{c|}{ Age group }} & \multicolumn{6}{c|}{ Year } \\
\cline { 2 - 7 } & $\mathbf{2 0 0 9}$ & $\mathbf{2 0 1 0}$ & $\mathbf{2 0 1 1}$ & $\mathbf{2 0 1 2}$ & $\mathbf{2 0 1 3}$ & $\mathbf{2 0 1 4}$ \\
\hline Children and Adolescent & 0.008 & 0.004 & 0.066 & 0.028 & 0.053 & 0.024 \\
\hline Young adults & 0.004 & 0 & 0.074 & 0.074 & 0.054 & 0.049 \\
\hline Old adults & 0.014 & 0.062 & 0.164 & 0.164 & 0.12 & 0.111 \\
\hline Elderly & 0.032 & 0.097 & 0.243 & 0.211 & 0.227 & 0.146 \\
\hline Total & 0.01 & 0.026 & 0.11 & 0.095 & 0.086 & 0.066 \\
\hline
\end{tabular}

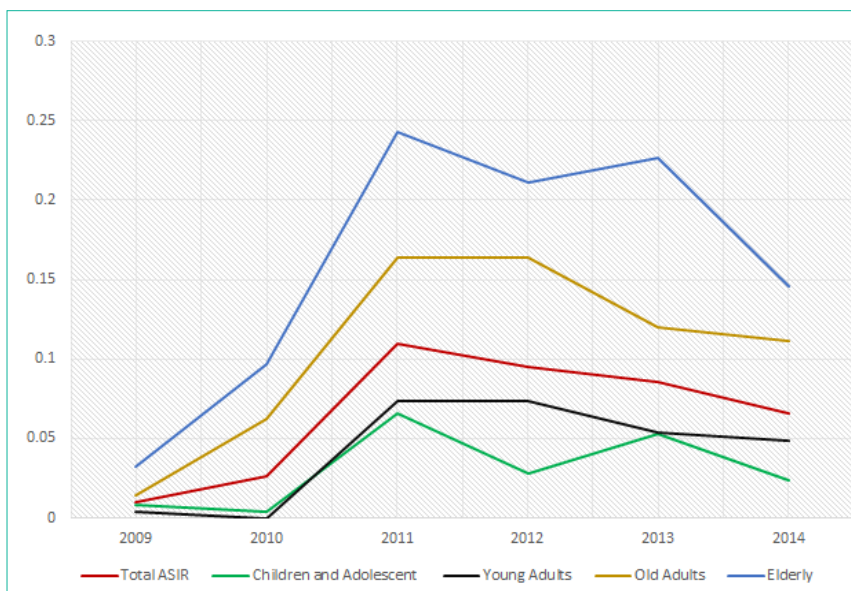

Figure 1: Annual trend of Age Specific Incidence Rate of CNS sarcomas. ASIR: Age Specific Incidence Rate

sarcomas are osteosarcoma, chondroblastoma, poorly differentiated round or spindle cell tumors, chordoma and neural sheet tumors [15]. Also, soft tissue sarcoma subtypes include liposarcoma, fibrosarcoma, leiomyosarcoma, rhabdomyosarcoma, angiosarcoma, gastrointestinal stromal tumor and undifferentiated sarcomas, along with other less frequent subtypes [16]. Although no exact mechanism or etiology is found to be responsible for development of sarcomas, several environmental and genetic mutations risk factors are considered to play important role in transformation of mesenchymal cells to different sarcoma subtypes. To give an instance, chromosomal translocation of chromosome 11 and chromosome 22 which results in EWS gene is associated with Ewing sarcoma [17]. Also, duplication of a part of chromosome 12 and resultant CDK4 gene and MDM2 gene 
Table 5: Morphological features of CNS sarcomas.

\begin{tabular}{|c|c|c|c|c|c|}
\hline Tumor type & Number & Tumor type & Number & Tumor type & Number \\
\hline Gliosarcoma & 63 & Small cell sarcoma, round cell & 4 & Alveolar soft part sarcoma & 1 \\
\hline $\begin{array}{l}\text { Desmoplastic small round cell } \\
\text { tumor }\end{array}$ & 33 & Rhabdomyosarcoma & 4 & Liposarcoma NOS & 1 \\
\hline Chordoma & 31 & Leiomyosarcoma & 3 & Myxosarcoma & 1 \\
\hline MPNST & 23 & Giant cell sarcoma & 3 & Fibrosarcoma & 1 \\
\hline Hemangiopericytoma malignant & 22 & Malignant fibrous histiocytoma & 3 & Endometrial stromal sarcoma & 1 \\
\hline Neurilemoma sarcoma & 13 & $\begin{array}{l}\text { MPNST with rhabdomyoblastic } \\
\text { differentiation }\end{array}$ & 2 & Mixed malignant tumor & 1 \\
\hline Malignant tumor spindle cell type & 12 & Malignant histiocytosis & 2 & Myxoid liposarcoma & 1 \\
\hline Ewing's sarcoma & 10 & Mixed type rhabdomyosarcoma & 2 & Embryonal rhabdomyosarcoma & 1 \\
\hline Spindle cells sarcoma & 9 & Synovial sarcoma & 2 & Glomus tumor malignant & 1 \\
\hline Meningeal sarcomatosis & 9 & Cerebellar sarcoma & 2 & $\begin{array}{l}\text { Fibrous mesothelioma malignant, Sarcomatoid } \\
\text { mesothelioma }\end{array}$ & 1 \\
\hline Chondrosarcoma & 5 & Osteosarcoma & 2 & & \\
\hline Malignant rhabdoid tumor & 5 & Epithelioid sarcoma & 1 & & \\
\hline Myeloid sarcoma & 5 & Mast cell sarcoma & 1 & & \\
\hline Schwannoma & 5 & Paraganglioma malignant & 1 & & \\
\hline Chondroid chordoma & 5 & Synovial sarcoma biphasic & 1 & & \\
\hline Sarcoma & 4 & Kaposi sarcoma & 1 & & \\
\hline
\end{tabular}

NOS: Not Otherwise Specified; MPNST: Malignant Peripheral Nerve Sheath Tumor.

play a significant role in development liposarcoma [18]. Furthermore, CSF1 gene which follows chromosomal translocation between chromosome 1 and chromosome 2, is related to increased risk of development of giant cell tumors of soft tissue [19]. Primary CNS sarcomas are primitive non-meningothelial tumors which represent about $0.2 \%$ of all intracranial malignancies, with equal trend to affect both sexes at any age [20-22]. Despite the trivial risk, sarcoma's burden on affected patients demands our fortified understanding of its natural history and pathogenesis to reach acceptable standards towards appropriate management of sarcomas individually. As stated above, mesenchymal origin of sarcomas provides emersion of wide variety of subtypes of both bone and soft tissue sarcomas [23]. Although rare, wide variety of presentation and subtypes have made Soft Tissue Sarcomas (STSs) a challenging malignancy with somehow unknown etiologic background.

In a report on 13 Children, Adolescent and Young Adults (CAYA) from 1990 to 2015, it is found that the affected patients with primary IS have mean age of 7 years, while another report on CAYA patient groups from 1990 to 2001, revealed the mean age of diagnosis of primary IS as 16.9 years $[24,25]$. In addition to the mentioned data of former report from United States of mean age of diagnosis, this 25-year observation stated unclassified sarcoma, followed by chondrosarcoma and rhabdomyosarcoma [24]. Also, Benesch et al conducted a cooperative study in Austria from 1988 to 2009 on 19 CAYA age group cases diagnosed with CNS sarcomas with mean age of 9.7 age with unspecified sarcoma was the most encountered histological type, followed by embryonal rhabdomyosarcoma, chondrosarcoma and malignant mesenchymal tumor [25]. Moreover, Al Ghahtany performed a similar study on 16 CAYA age group patients with CNS sarcoma with mean age of 4.8 and found 14 patients with intracranial sarcoma followed by the rest as spinal sarcomas in Canada [26]. Given the above results of mentioned researches, this study includes a considerable greater population of patients with
Table 6: CNS sarcomas distribution based on country's territories.

\begin{tabular}{|l|c|}
\hline \multicolumn{1}{|c|}{ Region of Iran } & Number of affected patients (Percentage) \\
\hline Central parts & $115(38.59 \%)$ \\
\hline Southern parts & $58(19.46 \%)$ \\
\hline Eastern parts & $53(17.78 \%)$ \\
\hline Western parts & $42(14.09 \%)$ \\
\hline Northern parts & $30(10.06 \%)$ \\
\hline Total & $298(100 \%)$ \\
\hline
\end{tabular}

age group 1 (0-14) which is comparable to CAYA age group (0-19), with similar first major subtype as unspecified sarcoma (22.5\%) to other two studies, followed by desmoplastic small round cell and gliosarcoma (12.9\% each) which demonstrated different sequence compared to previous surveys. Away from CAYA age group, Tihan et al reported a 20 -year survey on 43 cases diagnosed with primary CNS sarcomas with mean age of 40.3 years (3-75 years). In this study which was performed in United States and published in 2007, 16 cases were diagnosed with hemangiopericytoma, followed by 15 cases with chondrosarcoma and 3 with solitary fibrous tumor [27]. In addition, another 40-year report on 18 cases aged between 3-63 years (mean age 28) affected with primary IS revealed 15 cases with cerebral sarcoma, 2 with cerebellum involvement and one with spinal sarcoma [28]. Their histopathological evaluation exhibit fibrosarcoma, malignant fibrous histiocytoma and undifferentiated sarcoma as the most common tumor types in descending manner. Although Oliveira et al did not report detailed subtypes based on patient's age group, regarding their mean age of 28 years, our age group 2 (15-64) most common sarcoma types exhibit different sequence, as gliosarcoma (18.9\%) chordoma $11.8 \%$ and desmoplastic small round cell tumor (11\%). Although former surveys that were conducted on developed countries, demonstrates different sequence of common CNS sarcoma types based on their histopathologic features, authors believe that our 
results may act as a considerable representative of epidemiological and histopathological features of developing countries with different values compared to developed ones.

Despite scant available data on tumorigenesis of primary IS, studies suggest activation of receptor tyrosine kinase and angiogenesis pathways in most of the pediatric ISs [29,30]. Also, overexpression of platelet-derived growth factor receptor was found to be associated with osteosarcoma, rhabdomyosarcoma and Ewing sarcoma [31]. Beside special molecular and genetic processes responsible for development of this cancer, sarcomas are linked to multiple environmental offenders, such as ionizing radiation, alkylating agents and vinyl chloride and arsenic exposure. Moreover, sarcomas are associated with certain syndromes, including Li-fraumeni and neurofibromatosis type 1 [32,33]. Regarding primary IS, similar to other space occupying lesions, its clinical and neurological manifestation is consistent with tumor's location. Despite its natural tendency to originate from various cells in different locations, existing evidence suggest primary IS's predilection towards supra-tentorial regions, mostly affecting temporal and parietal lobes [34]. Neurological symptoms range from asymptomatic and mild headache, to significant neurological deficit and altered level of consciousness. However, multiple histopathological subtypes were found in previous conducted studies, being mostly reported as undifferentiated sarcomas, followed by chordoma, chondrosarcoma and rhabdomyosarcoma, as well as other less frequent subtypes [35-37]. Our analysis revealed obvious male to female dominance regarding prevalence of CNS sarcomas, with three quarters of the patients being in second age group category from 1564 years $(76.17 \%)$, followed by age group III (13.42\%) and I (10.4\%), showing higher tendency of CNS sarcomas to affect people away from their extremes of their ages. Our ASIR analysis revealed lower ASIR of CNS Sarcomas than expected number of cases compared to standard world population by WHO's ASIR as 0.035 person per 100000 -years. Also, our evidence suggests CNS sarcoma's trend to affect brain roughly 2 times more than spinal cord and isolated meningeal layers of less than 5\%. Also, WHO classification of CNS sarcomas of our studied patients revealed significant grade 9 or tumors of uncertain differentiation compared to other subtypes. Gliosarcoma represents the most frequent detected CNS sarcoma, followed by desmoplastic small round cell tumor, chordoma, malignant peripheral nerve sheet tumor and hemangiopericytoma malignant as top 5 lesions based on frequency. Moreover, our detailed report of patient's geographical distribution pattern may provide potential fields for environmental and public health activist to use this data as a primary reference in their future plans.

\section{Conclusion}

Coherent with various subtypes, multiple medical and surgical approaches have been suggested for patients diagnosed with primary IS. However, due to lack of unified viewpoint of its natural history and existence of criteria for selection of appropriate management approach, insufficiency of an optimal strategy towards primary IS, is perceived. This is the first report of epidemiological assessment of CNS sarcomas in Iran, which can act as a foundation for better understanding the underlying pathophysiological mechanism and selection of optimal treatment strategies based on improved understanding of these neoplasms.

\section{References}

1. Lahat G, Lazar A, Lev D. Sarcoma epidemiology and etiology: potential environmental and genetic factors. Surgical Clinics of North America. 2008; 88: 451-481.

2. Woods JS, Polissar L, Severson RK, Heuser LS, Kulander BG. Soft tissue sarcoma and non-Hodgkin's lymphoma in relation to phenoxyherbicide and chlorinated phenol exposure in western Washington. Journal of the National Cancer Institute. 1987; 78: 899-910.

3. Wang VY, Potts M, Chou D. Sarcoma and the spinal column. Neurosurgery Clinics of North America. 2008; 19: 71-80.

4. Johnson KJ, Carozza SE, Chow EJ, Fox EE, Horel S, McLaughlin CC, et al. Parental age and risk of childhood cancer: a pooled analysis. Epidemiology (Cambridge, Mass). 2009; 20: 475-483.

5. Thway K. Pathology of soft tissue sarcomas. Clinical oncology. 2009; 21 : 695-705.

6. Gerrand C, Athanasou N, Brennan B, Grimer R, Judson I, Morland B, et al. UK guidelines for the management of bone sarcomas. Clinical sarcoma research. 2016; 6: 7 .

7. Dyrop HB, Safwat A, Vedsted P, Maretty-Kongstad K, Hansen BH, Jørgensen $\mathrm{PH}$, et al. Characteristics of 64 sarcoma patients referred to a sarcoma center after unplanned excision. Journal of surgical oncology. 2016; 113: 235-239.

8. Vodanovich DA, Choong PF. Soft-tissue sarcomas. Indian journal of orthopaedics. 2018; 52: 35

9. Honoré C, Méeus P, Stoeckle E, Bonvalot S. Soft tissue sarcoma in France in 2015: epidemiology, classification and organization of clinical care. Journal of visceral surgery. 2015; 152: 223-230.

10. Mora J, Modak S, Cheung NK, Meyers P, De Alava E, Kushner B, et al. Desmoplastic small round cell tumor 20 years after its discovery. Future Oncology. 2015; 11: 1071-1081.

11. Yan D, Zhang J, Zhong D. Ewing's sarcoma in the spinal canal of T12 L3: A case report and review of the literature. Oncology Letters. 2019; 18: 61576163.

12. Merimsky O, Lepechoux C, Terrier P, Vanel D, Delord JP, LeCesne A. Primary sarcomas of the central nervous system. Oncology. 2000; 58: 210214.

13. Stiller CA, Trama A, Serraino D, Rossi S, Navarro C, Chirlaque MD, et al, RARECARE Working Group. Descriptive epidemiology of sarcomas in Europe: report from the RARECARE project. European Journal of Cancer. 2013; 49: 684-695.

14. Bekiesinska-Figatowska M, Duczkowska A, Duczkowski M, Bragoszewska $\mathrm{H}$, Romaniuk-Doroszewska A, Iwanowska B, et al. CNS Metastases from Bone and Soft Tissue Sarcomas in Children, Adolescents, and Young Adults: Are They Really So Rare?. BioMed research international. 2017; 2017: 1546473.

15. Skapek SX, Anderson J, Barr FG, Bridge JA, Gastier-Foster JM, Parham DM, et al. PAX-FOXO1 fusion status drives unfavorable outcome for children with rhabdomyosarcoma: A children's oncology group report. Pediatric blood \& cancer. 2013; 60: 1411-1417.

16. Missiaglia E, Williamson D, Chisholm J, Wirapati P, Pierron G, Petel F, et al. PAX3/FOXO1 fusion gene status is the key prognostic molecular marker in rhabdomyosarcoma and significantly improves current risk stratification. Journal of Clinical Oncology. 2012; 30: 1670-1677.

17. Italiano A, Di Mauro I, Rapp J, Pierron G, Auger N, Alberti L, et al. Clinical effect of molecular methods in sarcoma diagnosis (GENSARC): a prospective, multicentre, observational study. The Lancet Oncology. 2016; 17: 532-538.

18. Arumugasamy N. Some neuropathologic aspects of intracranial sarcomas. The Medical journal of Malaya. 1969: 169-173.

19. Harris MH, DuBois SG, Bender JL, Kim A, Crompton BD, Parker E, et al. Multicenter feasibility study of tumor molecular profiling to inform therapeutic decisions in advanced pediatric solid tumors: the individualized cancer therapy (iCat) study. JAMA oncology. 2016; 2: 608-615. 
20. Parsons DW, Roy A, Yang Y, Wang T, Scollon S, Bergstrom K, et al Diagnostic yield of clinical tumor and germline whole-exome sequencing for children with solid tumors. JAMA oncology. 2016; 2: 616-624.

21. Le Loarer F, Watson S, Pierron G, de Montpreville VT, Ballet S, Firmin N et al. SMARCA4 inactivation defines a group of undifferentiated thoracic malignancies transcriptionally related to BAF-deficient sarcomas. Nature genetics. 2015; 47: 1200 .

22. Dickson MA, D'Adamo DR, Keohan ML, D'Angelo SP, Carvajal RD, Gounder MM, et al. Phase II trial of gemcitabine and docetaxel with bevacizumab in soft tissue sarcoma. Sarcoma. 2015; 2015: 532478.

23. Verheijen P, Witjes $H$, Van Gorp J, Hennipman A, Van Dalen T. Current pathology work-up of extremity soft tissue sarcomas, evaluation of the validity of different techniques. European Journal of Surgical Oncology (EJSO). 2010; 36: 95-99.

24. Maher OM, Khatua S, Mukherjee D, Olar A, Lazar A, Luthra R, et al. Primary intracranial soft tissue sarcomas in children, adolescents, and young adults: single institution experience and review of the literature. Journal of neurooncology. 2016; 127: 155-163.

25. Postovsky S, Ash S, Ramu IN, Yaniv Y, Zaizov R, Futerman B, et al. Central nervous system involvement in children with sarcoma. Oncology. 2003; 65: 118-124.

26. Benesch M, von Bueren AO, Dantonello T, von Hoff K, Pietsch T, Leuschne I, et al. Primary intracranial soft tissue sarcoma in children and adolescents: a cooperative analysis of the European CWS and HIT study groups. Journal of neuro-oncology. 2013; 111: 337-345.

27. Al-Gahtany M, Shroft M, Bouffet E, Dirks P, Drake J, Humphreys R, et al. Primary central nervous system sarcomas in children: clinical, radiological, and pathological features. Child's Nervous System. 2003; 19: 808-817.

28. Tihan T, Griffin A, Ozuysal S. Primary sarcomas of the central nervous system: UCSF experience (1985-2005). Turkish J Pathol. 2007; 23: 5-15.

29. Oliveira AM, Scheithauer BW, Salomao DR, Parisi JE, Burger PC, Nascimento AG. Primary sarcomas of the brain and spinal cord: a study of 18 cases. The American journal of surgical pathology. 2002; 26: 1056-1063.
30. Abraham J, Prajapati SI, Nishijo K, Schaffer BS, Taniguchi E, Kilcoyne A McCleish AT, Nelon LD, Giles FG, Efstratiadis A, LeGallo RD. Evasion mechanisms to Igf1r inhibition in rhabdomyosarcoma. Molecular cancer therapeutics. 2011; 10: 697-707.

31. Rushing EJ, Armonda RA, Ansari Q, Mena H. Mesenchymal chondrosarcoma: a clinicopathologic and flow cytometric study of 13 cases presenting in the central nervous system. Cancer: Interdisciplinary International Journal of the American Cancer Society. 1996; 77: 1884-1891.

32. Chen X, Pappo A, Dyer MA. Pediatric solid tumor genomics and developmental pliancy. Oncogene. 2015; 34: 5207.

33. Shukla N, Ameur N, Yilmaz I, Nafa K, Lau CY, Marchetti A, et al. Oncogene mutation profiling of pediatric solid tumors reveals significant subsets of embryonal rhabdomyosarcoma and neuroblastoma with mutated genes in growth signaling pathways. Clinical Cancer Research. 2012; 18: 748-757.

34. Benesch M, von Bueren AO, Dantonello T, von Hoff K, Pietsch T, Leuschner I, et al. Primary intracranial soft tissue sarcoma in children and adolescents: a cooperative analysis of the European CWS and HIT study groups. Journal of neuro-oncology. 2013; 111: 337-345.

35. Weiss A, Gill J, Goldberg J, Lagmay J, Spraker-Perlman H, Venkatramani R, et al. Advances in therapy for pediatric sarcomas. Current oncology reports. 2014; 16: 395.

36. Kubo T, Piperdi S, Rosenblum J, Antonescu CR, Chen W, Kim HS, et al. Platelet-derived growth factor receptor as a prognostic marker and a therapeutic target for imatinib mesylate therapy in osteosarcoma. Cancer. 2008; 112: 2119-2129.

37. Yamaguchi SI, Ueki A, Sugihara E, Onishi N, Yaguchi T, Kawakami Y, et al. Synergistic antiproliferative effect of imatinib and adriamycin in plateletderived growth factor receptor-expressing osteosarcoma cells. Cancer science. 2015; 106: 875-882.

38. Ebrahimpour A, Chehrassan M, Sadighi M, Azizmohammad Looha M, Karim A, Akbari A, et al. The Survival and Incidence Rate of Ewing Sarcoma; a National Population-based Study in Iran (2008-2015). The Archives of Bone and Joint Surgery. 2020; 8: 391-399. 